\title{
On the effect of particle image intensity and image preprocessing on the depth of correlation in micro-PIV
}

\author{
Massimiliano Rossi • Rodrigo Segura • \\ Christian Cierpka $\cdot$ Christian J. Kähler
}

Received: 19 October 2010/Revised: 23 June 2011/Accepted: 19 August 2011/Published online: 9 September 2011

(C) The Author(s) 2011. This article is published with open access at Springerlink.com

\begin{abstract}
The depth of correlation (DOC) is an experimental parameter, introduced to quantify the thickness of the measurement volume and thus the depth resolution in microscopic particle image velocimetry ( $\mu$ PIV). The theory developed to estimate the value of the DOC relies on some approximations that are not always verified in actual experiments, such as a single thin-lens optical system. In many practical $\mu$ PIV experiments, a deviation of the actual DOC from its nominal value can be expected, due for instance to additional components present in the optical path of the microscope or to the use of image preprocessing before the PIV evaluation. In the presented paper, the effect of real particle image intensity distribution and image preprocessing on the thickness of the measurement volume is investigated. This is performed studying the defocusing of tracer particles and the DOC-related bias error present in $\mu$ PIV measurements in a Poiseuille flow. The analysis shows that the DOC predicted using the conventional formulas can be significantly smaller than its actual value. To overcome this problem, the use of an effective NA determined experimentally from the curvature of the image autocorrelations is proposed. The accuracy of this approach to properly predict the actual size of DOC is discussed and validated on the experimental data. The effectiveness of image preprocessing to reduce the DOC-related bias error is tested and discussed as well.
\end{abstract}

M. Rossi ( $)$ · R. Segura · C. Cierpka · C. J. Kähler Institute of Fluid Mechanics and Aerodynamics, Bundeswehr University Munich, 85577 Neubiberg, Germany e-mail: massimiliano.rossi@unibw.de

\section{Introduction}

Microparticle image velocimetry ( $\mu$ PIV) (Santiago et al. 1998) is nowadays a well-established, widely used technique to measure the fluid motion in microfluidic devices (Lindken et al. 2009). $\mu \mathrm{PIV}$ is a variation of the wellknown technique for macroscopic flows known as particle image velocimetry (PIV) (Adrian 1991). Both techniques derive the velocity from the measured displacement of tracer particles suspended in the fluid. The displacement is obtained from the cross-correlation of two subsequent digital images (image pair) of the particles in the flow, separated by a known time interval. The images are then divided in small regions, referred to as interrogation windows (IW), and the mean particle image displacement in each IW is determined by a cross-correlation of the two images.

One of the most critical points in the application of $\mu$ PIV, and main difference with macroscopic PIV, concerns the illumination of the particles. In standard PIV, a laser light sheet is generated to illuminate a region in the flow. The depth of field of the camera is typically larger than the thickness of the laser sheet, and therefore, the depth of the measurement plane is mainly defined by the thickness of the light sheet. To be more precise, however, the light sheet intensity distribution, the size of the particles and the camera sensitivity must also be taken into account (Raffel et al. 2007).

Creating a thin laser sheet is obviously very difficult, or not possible at all, in a device with micrometric dimensions. Therefore, in a $\mu$ PIV setup, the entire flow is illuminated. As a consequence of this, all the particles in the volume are illuminated, and the thickness of the measurement plane is defined by the depth of field of the microscope objective. In particular, in $\mu$ PIV experiments, 
this thickness is commonly expressed in terms of depth of correlation (DOC), defined as twice the distance from the object plane to the nearest plane in which a particle becomes sufficiently defocused so that it no longer contributes significantly to the cross-correlation analysis (Meinhart et al. 2000).

An analytical expression for the DOC was provided first by Olsen and Adrian (2000). Their analysis began by observing that the measured velocity in a certain position is given by a weighted average of the velocity in the interrogation volume:

$\mathbf{u}_{0}=\frac{\int \mathbf{u}(\mathbf{x}, t) W(\mathbf{x}) \mathrm{d} \mathbf{x}}{\int W(\mathbf{x}) \mathrm{d} \mathbf{x}}$

where $\mathbf{x}$ is the position vector in the flow, $\mathbf{u}$ the flow velocity and $W$ the weighting function. $W(\mathbf{x})$ has its maximum at the focal plane $(z=0$ being $z$ the coordinate parallel to the optical axis) and decreases as $z$ moves away from the focal plane. $W(\mathbf{x})$ can be used to set a distance $z_{\text {corr }}$ from the object plane beyond which the ratio $W(z) / W(0)$ falls below a threshold value $\varepsilon$, thus defining the DOC as 2 times $z_{\text {corr }}$.

Under the assumptions of (1) a single thin-lens system, (2) constant illumination, (3) small out-of-plane displacement, (4) particle image intensity distribution modeled as a Gaussian function and (5) $s_{o} \gg z$ with $s_{o}$ equal to the working distance of the lens, Olsen and Adrian (2000) were able to derive an analytical expression of the $W(z)$, which yields the well-known expression for the DOC:

$$
\mathrm{DOC}=2\left\{\frac{(1-\sqrt{\varepsilon})}{\sqrt{\varepsilon}}\left[f^{\# 2} d_{p}^{2}+\frac{5.95(M+1)^{2} \lambda^{2} f^{\# 4}}{M^{2}}\right]\right\}^{1 / 2}
$$

where $M$ and $f^{\#}$ are the magnification and f-number of the objective lens, respectively, $d_{p}$ the tracer particles' diameter, $\lambda$ the wavelength of the light and $\varepsilon$ the threshold value typically set to 0.01 .

A validation of Eq. 2 for different sets of particle sizes and objective lenses was provided by Bourdon et al. (2004a, b, 2006). In particular, the accuracy of the expression for $W(z)$ was tested, showing under ideal conditions, an excellent agreement between experimental and analytical data. Equation 2, eventually cited in slightly modified forms, is currently used to estimate the measurement depth in most $\mu$ PIV experiments.

However, some problems can arise in practical applications when more complex lens arrangements or specialized microscopes are present. In this case, some of the assumptions used to derive Eq. 2 are no longer valid, and the estimated DOC can significantly deviate from the actual DOC provided by the data. In particular, experiments carried out on shear flows suggested that in this condition, the actual DOC is larger than that predicted by Eq. 2. This was recently reported by Kloosterman et al. (2011) where $\mu$ PIV was used to estimate the flow rate in capillaries using large DOC $\mu$ PIV. They showed that the difference between estimated and actual DOC can lead to an underestimation of the maximum velocity of up to $25 \%$. An additional problem to correctly estimate the actual DOC in practical applications is introduced by the image preprocessing that is widely used to eliminate background noise or defocused particles.

This paper intends to address this issue, i.e., what is the actual size of the DOC in a practical $\mu$ PIV experiment in which the tracer particles are imaged using a conventional microscope, with non-ideal conditions, and image preprocessing is applied to the images. To do this, a series of tests were performed using a Zeiss Axio observer Z1 inverted microscope. In particular, the actual $W(z)$ for different sets of particle sizes and objective lenses was measured following the procedure used by Bourdon et al. (2004a, b), and the actual size of the DOC was estimated by looking at the bias error of velocity measurements in a Poiseuille flow inside a microchannel with a rectangular cross-section. The effect of particle image preprocessing was studied as well.

The paper is structured as follows: in Sect. 2, some theoretical aspects related to the analytical expression of the DOC will be revised and discussed; in Sect. 3, the experimental setup used will be described; in Sect. 4, the results of the experiments will be presented and discussed. In the conclusions, the outcome of the experiments will be summarized, guidelines to correctly estimate the DOC in a general $\mu$ PIV experiment will be proposed, and the possibility to use image preprocessing in order to significantly reduce the bias error arising from the DOC will be discussed.

\section{Theoretical framework}

The DOC formula is often cited in research and review articles with slightly different formulations. The modifications are mostly due to two reasons: the $f^{\#}$ is substituted by the numerical aperture NA, which is used in microscopy more often, and the DOC value is adjusted to account for the effect of refraction, which modifies the measurement depth when the immersion medium of the lens (typically air or oil) is different from the one of the working fluid (typically water). The different approaches chosen to overcome these problems can lead to different estimated values of the DOC for the same setup that can considerably diverge (in some cases by more than 50\%) from one another.

Concerning the $f^{\#}$ for instance, Bourdon et al. (2006) suggested that it be substituted by $n_{0} /(2 \mathrm{NA})$, while 
Meinhart and Wereley (2003) defined an infinity corrected $f$-number $\left(f^{\# \infty}\right)$ for infinity-corrected microscopic systems. To account for refraction, Bourdon et al. (2004a, b) used the ratio $n_{w} / n_{0}$ (with $n_{w}$ being the refraction index of the fluid and $n_{0}$ the refraction index of the lens immersion medium), Rossi et al. (2010) used the ratio based by the exact refraction of marginal rays, Meinhart and Wereley (2003) suggested that the change in refraction index produces an effective NA, different from the NA of the lens.

In this section, the theory will be briefly revised and discussed, and a consistent formulation for the DOC will be proposed.

\subsection{Particle image diameter}

The analytical model used to derive the DOC formula in (2) assumes that the intensity distribution $\hat{J}_{0}$ of a particle image can be modeled as a Gaussian function (Olsen and Adrian 2000). This can be generally written as:

$\hat{J}_{0}[r, z]=\frac{A}{d_{e}^{2}(z)\left(s_{o}+z\right)^{2}} \exp \left(\frac{-4 \beta^{2} r^{2}}{d_{e}^{2}(z)}\right)$

where $r$ is the distance from the particle center, $d_{e}$ the particle image diameter and $A$ and $\beta$ two constants. The particle image diameter $d_{e}$ corresponds to the point where the intensity distribution falls below a certain threshold, given by $\exp \left(-\beta^{2}\right)$ times the maximum intensity at the center. The value of $\beta^{2}$ is typically equal to 3.67 (Adrian and Yao 1985).

The particle image diameter $d_{e}$ is the result of three contributions: one due to the geometrical dimensions of the particle, one due to diffraction and one due to the distance $z$ of the particle from the focal plane (defocusing). Under the assumption that these three contributions can be modeled as Gaussian functions, the resulting particle image diameter is given by (Olsen and Adrian 2000):

$d_{e}=\left(M^{2} d_{p}^{2}+d_{s}^{2}+d_{f}^{2}\right)^{1 / 2}$

The single thin-lens optical geometry depicted in Fig. 1 was used by Olsen and Adrian (2000) to derive the particle image diameter. The lens corresponds to the objective lens of the microscope, $D_{a}$ is the objective lens' diameter and $s_{o}$ is the working distance of the lens.

The particle geometrical dimension term in Eq. 4 is simply obtained by the particle diameter $d_{p}$ times the magnification of the system. The diffraction term is given by the diameter of the point response system of a diffraction-limited lens (Adrian and Yao 1985), given by:

$d_{s}=2.44(M+1) \lambda f^{\#}$

Considering the definition of $f^{\#}=f / D_{a}$, the expression of $M=s_{i} / s_{o}$ and the Gaussian lens formula:

$\frac{1}{s_{o}}+\frac{1}{s_{i}}=\frac{1}{f}$

Eq. 5 can be rewritten as:

$d_{s}=2.44 M \lambda \frac{s_{o}}{D_{a}}$

From trigonometry, we have that:

$\frac{s_{o}}{D_{a}}=\frac{1}{2}\left(\frac{1}{\sin ^{2} \theta}-1\right)^{1 / 2}$

so substituting Eq. 8 in 7 and using the definition of $\mathrm{NA}=n_{0} \sin \theta$, results in the diffraction term as a function of NA:

$d_{s}=1.22 M \lambda\left(\frac{n_{0}^{2}}{\mathrm{NA}^{2}}-1\right)^{1 / 2}$

This expression, derived for a general case, coincides with the one derived by Meinhart and Wereley (2003) for infinity corrected systems.

The defocusing term was derived using geometrical optics for the single thin-lens configuration and is given by:

$d_{f}=\frac{M z D_{a}}{s_{o}+z}$

Assuming $s_{o} \gg z$ and substituting Eqs. 8, 9 and 10 in 4, the particle image diameter as a function of $z$ and NA is given by the following expression:
Fig. 1 Optical geometry used in deriving the particle image diameter

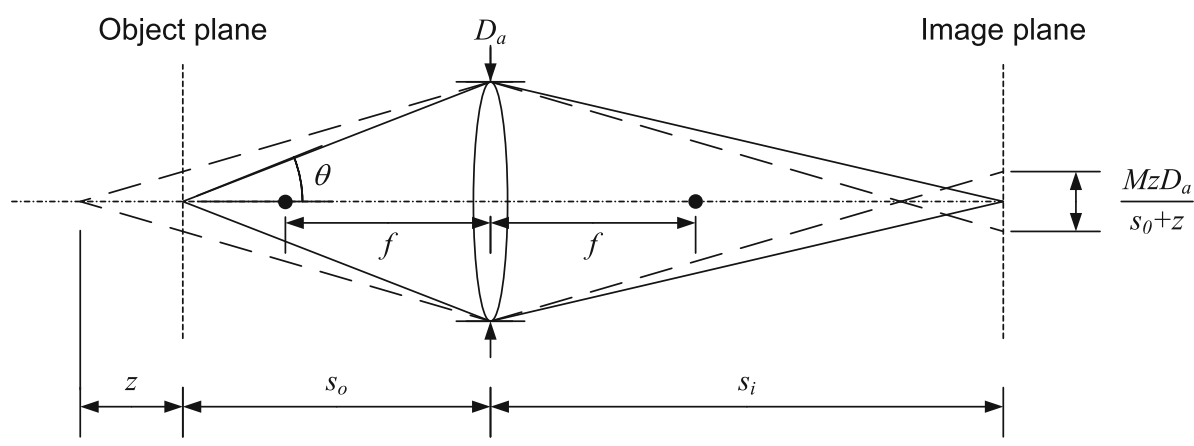


Fig. 2 Effect of refraction

(a)

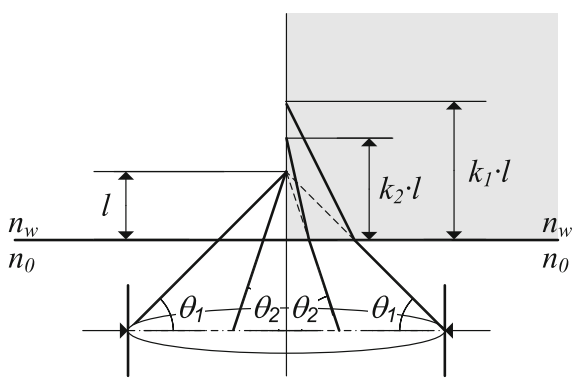

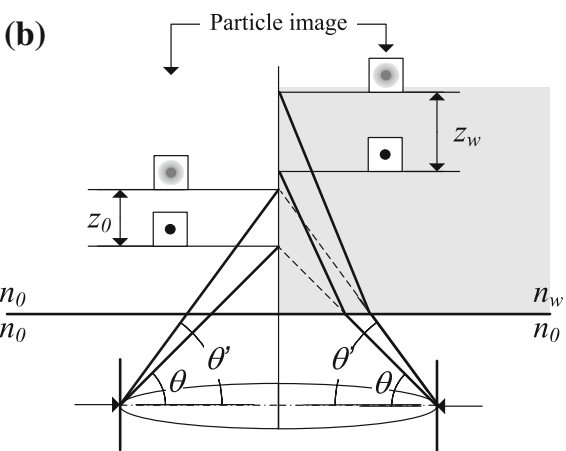

$d_{e}=M\left[d_{p}^{2}+1.49 \lambda^{2}\left(\frac{n_{0}^{2}}{\mathrm{NA}^{2}}-1\right)+4 z^{2}\left(\frac{n_{0}^{2}}{\mathrm{NA}^{2}}-1\right)^{-1}\right]^{1 / 2}$

\subsection{Effect of refraction}

When the refraction index of the immersion medium of the lens $n_{0}$ is different from the one of the working fluid $n_{w}$, there will be a deflection of the rays at the interface due to refraction. A ray is deflected depending on its angle of incidence according to Snell's law: $n_{0} \sin \theta_{0}=n_{w} \sin \theta_{w}$. This means that a ray that would reach its focal plane at a distance $l$ from the interface when $n_{0}=n_{w}$, will reach it at a distance of $k \cdot l$ when $n_{0} \neq n_{w}$. The factor $k$ depends on the incidence angle $\theta$ as shown in Fig. 2.a and can be determined from Snell's law and trigonometry:

$k(\theta)=\frac{n_{w}}{n_{o}} \frac{\left(1-\frac{n_{o}^{2}}{n_{w}^{2}} \sin ^{2} \theta\right)^{1 / 2}}{\left(1-\sin ^{2} \theta\right)^{1 / 2}}$

Determining the position and shape of the focal plane after refraction is not straightforward and depends on the design of the real lens, which often presents corrections for aberrations. Hereby, we will make the assumption that the entire focal plane remains planar and is only shifted by a factor $k(\theta)$ corresponding to the ray with inclination $\theta$.

The shift of the focal plane has to be taken into account when one determines the defocusing of a particle image. Without refraction, the defocusing term for a particle at a distance $z_{0}$ from the focal plane is given by Eq. 10. When refraction is present, the same defocusing is obtained at the distance $z_{w}$ from the focal plane, which can be determined from geometry (see Fig. 2b):

$z_{w}=\left(l+z_{0}\right) \cdot k\left(\theta^{\prime}\right)-l \cdot k(\theta)$

For $z_{0} \ll s_{o}$, we can assume $\theta \approx \theta^{\prime}$ and $z_{w}=k(\theta) \cdot z_{0}$. Now, it remains to be determined which $\theta$ must be used to calculate the shift of the focal plane. In this case, an experimental approach was used to determine the $k$ of lenses with different NA. The microscope was initially focused on particles that were stuck to the bottom wall of the microchannel using the selected lens. The microscope stage was then moved until the focal plane of the microscope was displaced to the particles stuck on the top wall. The focal position was determined by maximizing a focusing sharpness function. The variance of the pixel gray level of the image was used as the focusing sharpness function (Yeo et al. 1993).

The displacement of the microscope stage from one focal position to the other changes depending on the medium inside the channel. If the channel is filled with the immersion medium of the lens (for the lenses used in this work, this medium was always air), the displacement is equal to the actual height of the channel. If the channel is filled with water, the displacement is equal to the height of the channel divided by $k$ as explained earlier.

The graph in Fig. 3 shows the theoretical values of $k$ for the axial rays $(\sin \theta=0)$ and the marginal rays $(\sin \theta=$ $\left.\mathrm{NA} / n_{0}\right)$ as a function of the NA of the lens, and the experimental value of $k$, determined for 4 types of lenses with NAs, respectively, equal to $0.3,0.4,0.6,0.75$. As can be observed in the graph in Fig. 3, the experimental $k$ is in

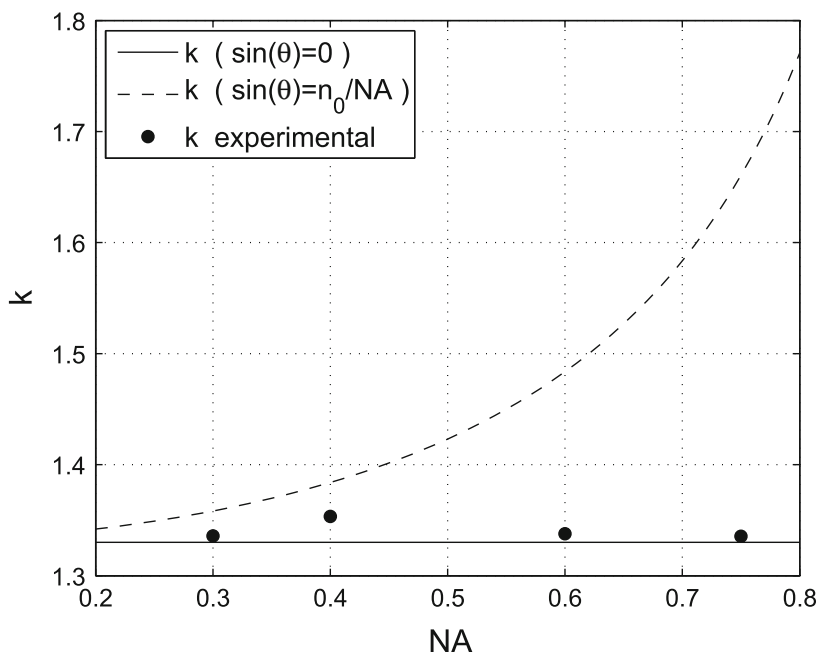

Fig. 3 Theoretical values of $k$ as a function of the NA for axial rays $(\sin \theta=0)$ and marginal rays $\left(\sin \theta=\mathrm{NA} / n_{0}\right)$. Experimental values of $k$ determined for 4 types of lenses with NA, respectively, equal to $0.3,0.4,0.6,0.75$ 
Fig. 4 Schematic of lens arrangement of a microscope with infinity corrected lens in its basic configuration

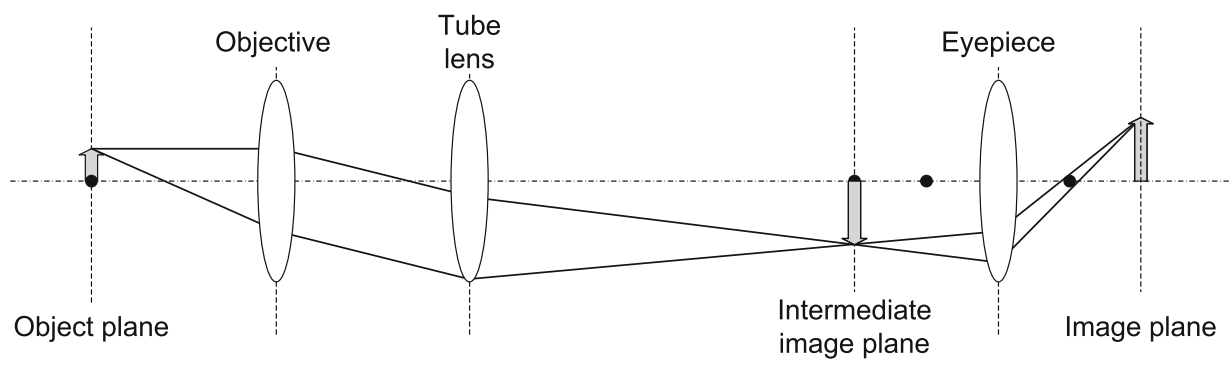

good agreement with the theoretical value for the axial rays, corresponding to the expression $k=n_{w} / n_{0}$. This result might be different when different types of lenses are used; therefore, it is advisable to experimentally characterize this parameter for each experimental setup.

\subsection{Depth of correlation formula}

Bourdon et al. (2004a, b) showed that the weighting function in (1) for a certain depth coordinate $z_{k}$ is equal to the curvature of the local correlation function evaluated at the local maximum:

$W\left(z_{k}\right)=\frac{\partial^{2}\left\langle R_{D}\right\rangle_{k}}{\partial^{2} s^{2}}$

Under ideal conditions, this reduces to (Olsen and Adrian 2000):

$W(z)=\frac{d_{e}^{4}(0)}{d_{e}^{4}(z)}$

The DOC formula is obtained solving for $z_{\text {corr }}$ the following equality: $W\left(z_{\text {corr }}\right)=\varepsilon$. Using Eqs. 11 and 15 yields:

$$
\begin{aligned}
\mathrm{DOC}= & \frac{n_{w}}{n_{0}}\left\{\frac{(1-\sqrt{\varepsilon})}{\sqrt{\varepsilon}}\left(\frac{n_{0}^{2}}{\mathrm{NA}^{2}}-1\right)\right. \\
& \left.\times\left[d_{p}^{2}+1.49 \lambda^{2}\left(\frac{n_{0}^{2}}{\mathrm{NA}^{2}}-1\right)\right]\right\}^{1 / 2}
\end{aligned}
$$

The multiplicative factor $n_{w} / n_{0}$ is introduced to account for refraction. This formulation will be adopted in the following sections of the paper to obtain the theoretical values of the DOC.

\subsection{Real microscope system}

In the most practical applications, the $\mu \mathrm{PIV}$ measurements are performed in commercial inverted or upright microscopes. A microscope, in its basic configuration, is composed of a 2-lens optical system: an objective that focuses an image in an intermediate image plane and an eyepiece that looks at that image. When human eyes are used to observe a specimen through the microscope, the eyepiece is a negative lens that forms a virtual image in front of the lens. When a CCD camera is used, the eyepiece is a positive lens that projects the intermediate image in the CCD. Quite often, infinity corrected lenses are also adopted to allow for the introduction of auxiliary components such as filters or polarizer (Fig. 4). Additional optical elements can be present as well to account for aberrations, to provide optical zoom and so on.

As a consequence of the increased complexity of a real microscope in comparison with the single thin-lens model adopted to derive the DOC formula, the following observations can be made:

- The presence of additional optical components or aperture stops placed down the optical path can make the final NA of the system smaller than the one declared by the manufacturer of the objective lens.

- The rate of defocusing in Eq. 10, derived from the optical geometry of a single thin-lens model, might fail to predict the actual rate of defocusing of more complex systems.

- Equation 11 is symmetric to the focal plane under the assumption that $s_{o} \gg z$. This might not be the case for high NA lenses, resulting in an asymmetric defocusing pattern.

- Multiple lens arrangements can also lead to an asymmetric defocussing. For instance, the infinity corrected lens system in Fig. 4 is designed to have parallel rays between the objective and the tube lens. However, this holds only for in-focus images, whereas for out-offocus images, the rays are converging or diverging depending on the direction of defocusing. This might result in a reduction of the NA in only one defocus direction, in the case that the diverging rays start to exceed the aperture of the tube lens.

- Additional nonlinear optical distortions, especially at higher NA, occur at the outer regions of the CCD sensor.

To properly address these issues, it would be necessary to know exactly the configuration of the microscopic system in use, which is often very difficult or not possible at all in case of proprietary commercial microscopes. In this 
paper, we suggest to account for these effects using an effective NA defined as:

$\mathrm{NA}_{\mathrm{eff}}=C_{d} \mathrm{NA}$

where $C_{d}$ is a constant to be determined experimentally. This approach has the advantage to be extremely simple and does not require any change in the DOC formula. On the other hand, it does not account for the effect of asymmetries or more complex defocusing or distortion patterns. The accuracy of this approach will be tested and discussed in the following sections.

\section{Experimental setup}

The experimental particle images used for the comparison with the theory were obtained by taking images of tracer particles of different sizes stuck to the top wall of a microchannel filled with distilled water. The bottom wall of the microchannel was a 0.17 -mm-thick glass plate that also served as optical access. Polystyrene latex particles, fabricated by Microparticles $\mathrm{GmbH}$ and coated with a red fluorescent dye, were used. Four particle sizes, with mean diameters, $d_{p}$, of $0.5 \mu \mathrm{m}, 1 \mu \mathrm{m}, 2 \mu \mathrm{m}$ and $5 \mu \mathrm{m}$ were tested. Images were taken using an Axio observer Z1 inverted microscope manufactured by Carl Zeiss, coupled with a two cavity frequency-doubled Litron Nano $\mathrm{S}$ Nd:Yag laser $(532 \mathrm{~nm})$ as illumination source. Four different Zeiss EC-Neofluar objective lenses were used with magnifications of $10 \times, 20 \times, 40 \times$ and $63 \times$, and respective NA of $0.3,0.4,0.6,0.75$. A 12-bit, $1,280 \times 1,024$ pixels, interline transfer CCD camera (PCO Sensicam) was used for the image acquisition.

The same system was used to perform $\mu$ PIV measurements in a microchannel with a cross-section of $200 \times 500 \mu^{2}$, where a pressure driven Poiseuille flow was established. The flow was generated by pushing homogeneously seeded distilled water through the channel using a neMESYS precision syringe pump, manufactured by Cetoni $\mathrm{GmbH}$, at a rate of $10 \mathrm{ml} / \mathrm{hr}$. The PIV measurements were repeated at the same flow conditions using the different combinations of tracer particle diameter and objective lens described above. The $\mu$ PIV system was installed on an optical table to damp vibrations. Recordings and image preprocessing were made using the DaVis 7.4 software package from LaVision. The velocity vectors result from a correlation averaging of 1000 images in each measurement plane, performed with a self-developed Matlab code. The size of the interrogation window was adapted to account for different magnifications and was $64 \times 64$ pixels for $M=20 \times, 128 \times 128$ pixels for $M=40 \times$ and $202 \times 202$ pixels for $M=63 \times$. Image preprocessing was performed by subtracting a sliding minimum over time to reduce non-uniformities and backreflections followed by an intensity histogram filter to highlight in focus particles and remove highly defocused ones.

\section{Results}

\subsection{Particle defocusing and weighting function}

The images of defocused particles can give a first qualitative impression of the behavior of a real microscopic system. Particle images obtained from observing a $2-\mu \mathrm{m}$ particle with $M=20 \times$ and $M=63 \times$ lenses, at different distances from the focal plane, are shown in Fig. 5. The corresponding theoretical diameters calculated with Eq. 11, represented by a white circle, are superimposed on each image. From a qualitative observation of the images, the theoretical diameters match the border of the particle images at $M=20 \times$ but fail for images at $M=63 \times$.

To quantitatively address the problem, the particle image diameter has to be determined experimentally. Unfortunately, this approach cannot be applied to real images in a straightforward manner for several reasons. A real defocused particle image, specially at large magnifications and NAs, starts to stray from the Gaussian behavior, showing, for instance, the Airy patterns. Moreover, background noise is present in every image, which makes the unambiguous determination of the threshold problematic. Additionally, for small particles at small magnification, the particle image size is reduced to a few pixels, and the reconstruction of the intensity distribution function from a single image is problematic. A more robust diameter determination can be obtained from the image autocorrelation.

The measured diameters for the two cases in Fig. 5 are reported in Fig. 6 for different depth positions, together with the maximum image intensity and the corresponding $d_{e}(z)$ calculated using (11). The diameters were measured by setting a threshold equal to 0.15 between the background noise level and the peak intensity of the image autocorrelation. The diameter values were divided by a factor $\sqrt{2}$ to account for stretching in the autocorrelation space (this is exact strictly for Gaussian-like distributions of particle image intensities). It can be observed that for $M=20 \times$ the measured diameters follow the theoretical prediction, while in the case of $M=63 \times$ the diameters grow much more slowly than expected. Moreover, for $M=63 \times$, a clear asymmetric behavior can be observed, in particular looking at the drop of maximum intensity that is much sharper moving to positive $z$.

The results in Figs. 5 and 6 show that the analytical model in (11) is inadequate to predicts the particle image diameter for the $M=63 \times$ objective and suggest that the 
Fig. 5 Particle images obtained observing a $2-\mu \mathrm{m}$ particle with $M=20 \times$ and $M=63 \times$ lenses, at different distances from the focal plane. The corresponding theoretical diameters for each image, obtained from Eq. 11, are represented with a superimposed white circle
Fig. 6 Particle image diameters (left $y$-axis) and maximum particle image intensity (right $y$-axis) as a function of the distance $z$ from the focal plane $(z=0)$, for a $2-\mu \mathrm{m}$ particle observed with $M=20 \times$ and $M=63 \times$ lenses

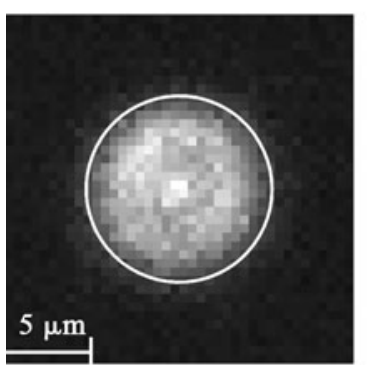

$Z=-11.8 \mu \mathrm{m}$

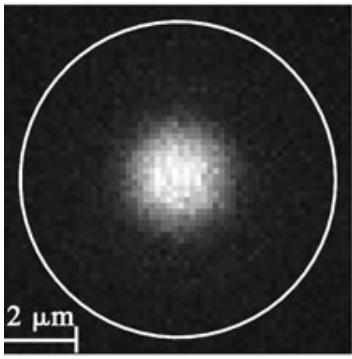

$Z=-3.7 \mu \mathrm{m}$
$M=20 \mathrm{x} \quad N A=0.4 \quad d_{p}=2 \mu \mathrm{m}$

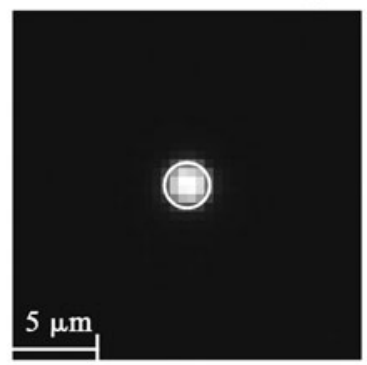

$z=0 \mu \mathrm{m}$

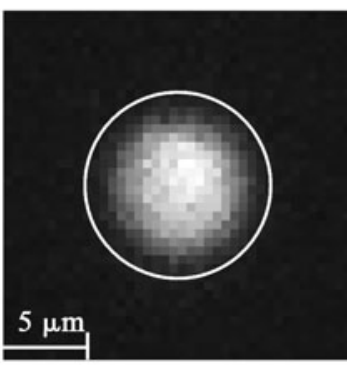

$Z=11.8 \mu \mathrm{m}$

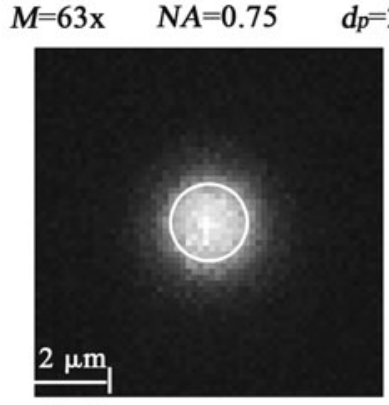

$Z=0 \mu \mathrm{m}$

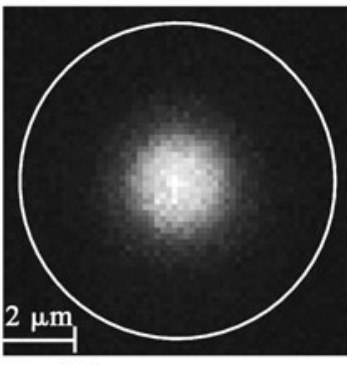

$z=3.7 \mu \mathrm{m}$
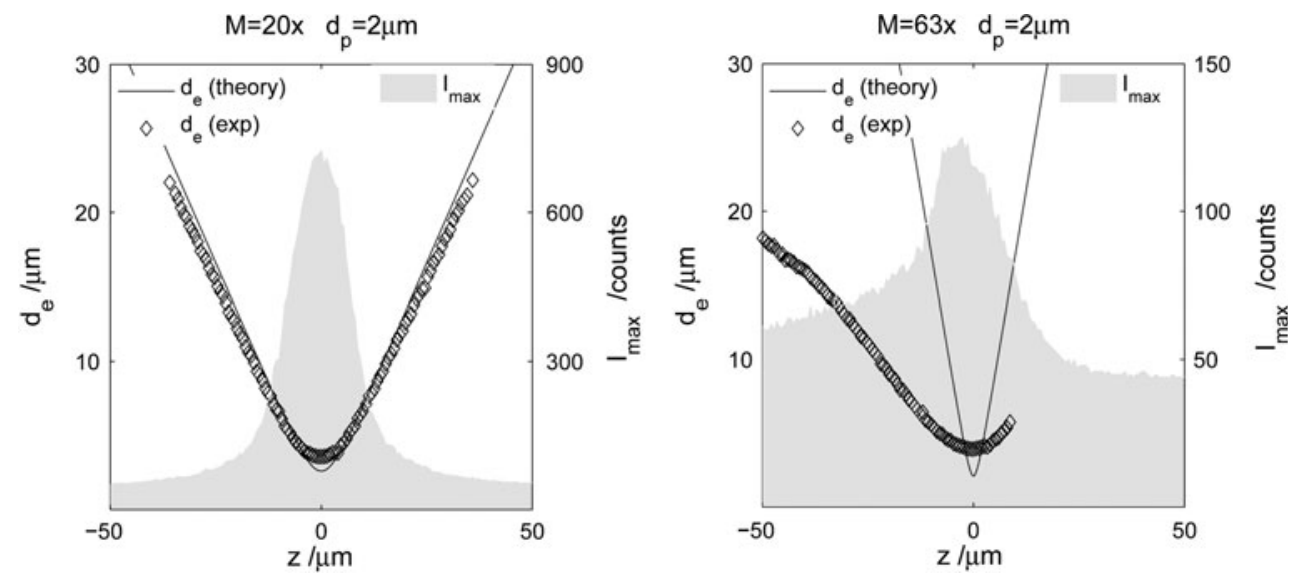

DOC predicted by (16) will be biased as well. However, to properly evaluate the contribution of real defocused particle images to the DOC prediction, one needs to measure the curvature of the image autocorrelation (i.e., the weighting function $W$ ), as shown by Bourdon et al. (2004a, b). The measured $W$ at different $z$ positions for the two cases in Fig. 5 is plotted in Fig. 7. In the same graphs are also plotted the $W$ obtained from synthetic images, the $W$ obtained using the theoretical model ( $W_{\text {th }}$, dotted line), and the $W$ obtained using the theoretical model in which the effective numerical aperture $\mathrm{NA}_{\text {eff }}$ was used ( $W_{\text {eff }}$, continuous line). For the case of $M=20 \times$, the theoretical model slightly underestimates the width of the actual $W$, and a practically perfect match can be obtained using a $\mathrm{NA}_{\text {eff }}$ with $C_{d}=0.75$. For the case of $M=63 \times$, the theoretical model strongly underestimate the width of the actual $W$, and an asymmetric behavior can be observed moving away to the focal plane. However, the asymmetric part is restricted to regions in which $W<0.4$, and a good fit with the theoretical model can be achieved in the central part using a NA eff with $C_{d}=0.37$.

Following this approach, the curvature of the image autocorrelation at the different distances from the object plane was determined for each combination of lenses and particle sizes reported in Sect. 3, apart from the case of $d_{p}=0.5 \mu \mathrm{m}$ observed with $M=10 \times$, for which the particle images were too small to be properly resolved. The data are presented in the graphs in Fig. 8. Each graph shows the measured $W$ for a given configuration together with the $W_{\text {eff }}$ calculated using the corresponding $\mathrm{NA}_{\text {eff }}$. The $x$-axis, showing the distance $z$ from the focal plane, was normalized over the corresponding DOC calculated 

weighting function $W(z)$ calculated using: (1) real particle images, (2) synthetic particle images, (3) Eq. 15 with the nominal NA, (4) Eq. 15 with the effective NA. Data shown for a $2-\mu \mathrm{m}$ particle observed with $M=20 \times$ and $M=63 \times$ lenses
Fig. 7 Comparison of the
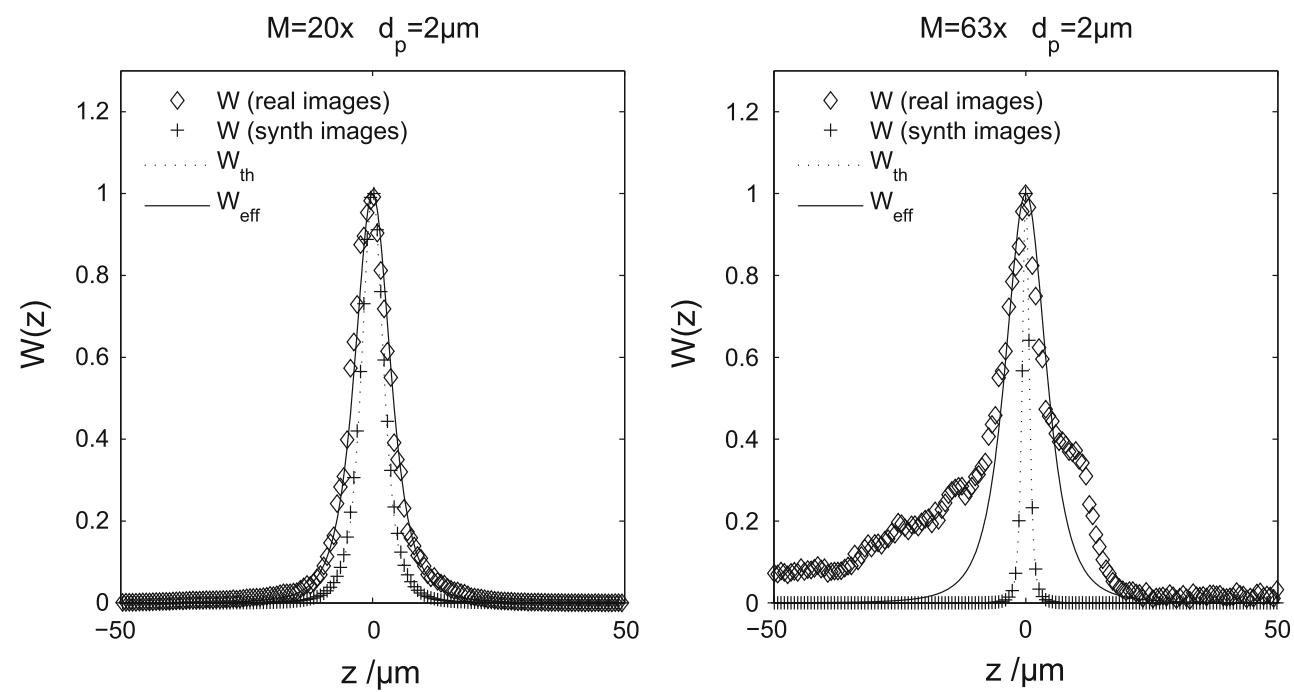

Fig. 8 Weighting function $W$ measured at different distances from the focal plane for different combinations of lenses and particle diameters
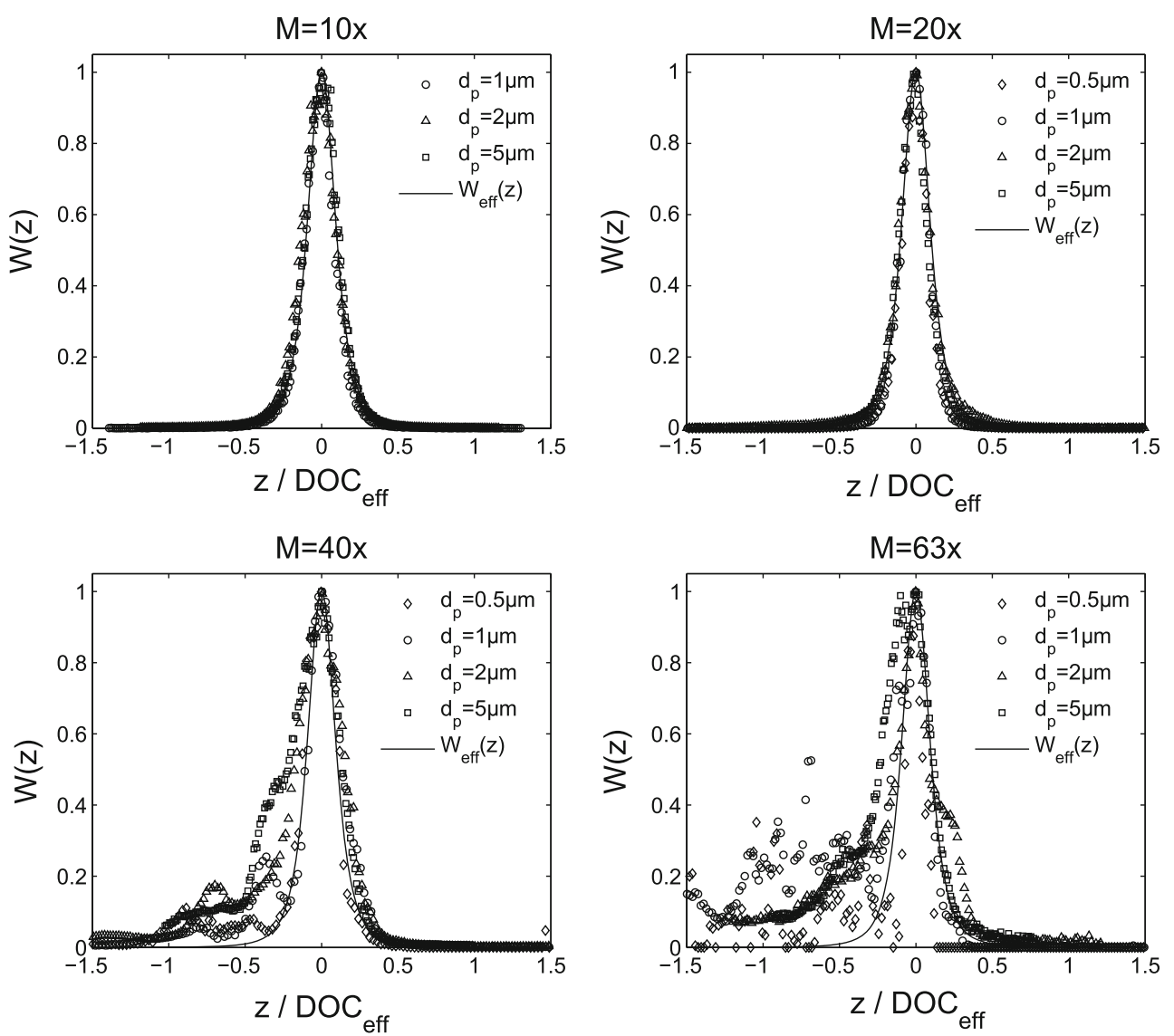

using $\mathrm{NA}_{\text {eff }}\left(\mathrm{DOC}_{\text {eff }}\right)$, so that the $W_{\text {eff }}$ was always represented by the same function. The parameter $C_{d}$ was derived experimentally for each lens searching the value that provided the best fit with the experimental data. The $C_{d}$ values for the different configurations together with the DOC values obtained from the nominal NA (denoted as $\mathrm{DOC}_{\mathrm{th}}$ ) and the effective NA (denoted as $\mathrm{DOC}_{\mathrm{eff}}$ ), are reported in Table 1.
For magnifications of up to $20 \times$ (and NA up to 0.4), the model using $\mathrm{NA}_{\text {eff }}$ is able to precisely predict the particle image diameter. For the $40 \times$ lens, the experimental curves start to deviate from the theory for negative $z$; however, a good match between experiments and theory is observed in the central part. The same behavior can be also observed for the $63 \times$ lens except for the largest particles with $d_{p}=5 \mu \mathrm{m}$. In this case, the width of $W$ is significantly 
Table 1 Theoretical and effective DOC for different combination of lenses and particle sizes

\begin{tabular}{|c|c|c|c|c|c|c|c|c|}
\hline \multirow{2}{*}{$d_{p}(\mu \mathrm{m})$} & \multicolumn{2}{|l|}{$10 \times / 0.3$} & \multicolumn{2}{|l|}{$20 \times / 0.4$} & \multicolumn{2}{|l|}{$40 \times / 0.6$} & \multicolumn{2}{|l|}{$63 \times / 0.75$} \\
\hline & $\mathrm{DOC}_{\mathrm{th}}(\mu \mathrm{m})$ & $\begin{array}{l}\mathrm{DOC}_{\mathrm{eff}}(\mu \mathrm{m}) \\
\left(C_{d}=0.80\right)\end{array}$ & $\mathrm{DOC}_{\mathrm{th}}(\mu \mathrm{m})$ & $\begin{array}{l}\mathrm{DOC}_{\text {eff }}(\mu \mathrm{m}) \\
\left(C_{d}=0.75\right)\end{array}$ & $\mathrm{DOC}_{\mathrm{th}}(\mu \mathrm{m})$ & $\begin{array}{l}\mathrm{DOC}_{\text {eff }}(\mu \mathrm{m}) \\
\left(C_{d}=0.52\right)\end{array}$ & $\mathrm{DOC}_{\mathrm{th}}(\mu \mathrm{m})$ & $\begin{array}{l}\mathrm{DOC}_{\text {eff }}(\mu \mathrm{m}) \\
\left(C_{d}=0.37\right)\end{array}$ \\
\hline 0.5 & - & - & 15.7 & 29.6 & 5.7 & 27.1 & 2.8 & 34.9 \\
\hline 1.0 & 31.5 & 49.4 & 17.6 & 31.5 & 7.4 & 29.1 & 4.2 & 36.9 \\
\hline 2.0 & 38.4 & 56.8 & 23.6 & 38.4 & 11.8 & 35.9 & 7.4 & 44.0 \\
\hline 5.0 & 69.7 & 93.2 & 48.1 & 69.7 & 27.1 & 66.7 & 17.7 & 77.1 \\
\hline
\end{tabular}

larger also in the central part, and the only use of an effective NA in the model is not sufficient to correctly predict the trend of $W$ also at short distances from the focal plane. Besides this last configuration (that is however never used in practical applications), the theoretical model combined with an effective NA, experimentally determined, provides a good estimate of the weighting function in proximity of the focal planes for all the different tested configurations and can be used to determine the actual size of the DOC in the experiment.

The results show that the actual DOC is indeed larger than the one predicted using the nominal NA of the objective lenses, especially when high NA lenses are used. This is evident in the graph in Fig. 9, where the ratio between $\mathrm{DOC}_{\mathrm{eff}}$ and $\mathrm{DOC}_{\mathrm{th}}$ as a function of the NA and the $d_{p}$ is presented. For NA up to 0.4 , the $\mathrm{DOC}_{\text {eff }}$ is always approximately between 1.3 and 2 times the $\mathrm{DOC}_{\mathrm{th}}$. For $\mathrm{NA}=0.6$, the $\mathrm{DOC}_{\text {eff }}$ is approximately 3-5 times larger than the $\mathrm{DOC}_{\mathrm{th}}$. For $\mathrm{NA}=0.6$, this ratio ranges between 4 and 12. The largest errors are observed for large NAs and small $d_{p}$ s.

To conclude this subsection, it must be added that all the results were obtained taking particle images in the central region of the $\mathrm{CCD}$ sensor. Particle images taken at the outer regions of the sensor, especially at higher NA, might suffer for nonlinear distortions that were not taken into account in this analysis. Since this paper intends to provide a general single value of DOC in real experiments, it was chosen to limit the investigation to the central region of the CCD sensor. Moreover, preliminary experiments suggested that this aspect is negligible for the lenses used in this investigation. However, it is worth to keep in mind this aspect when measurements with high NA objectives are planned on the entire CCD area.

\subsection{Velocity measurement}

A direct consequence of the DOC is a bias error in velocity measurements taken in a flow where velocity gradients in the $z$-direction are present (Olsen 2009). For instance, if velocity measurements are taken scanning through a microchannel in which a Poiseuille flow is established, the reconstructed velocity profile will present a systematic error due to the DOC: in the region close to the center of the channel, the velocity is underestimated due to contribution of particles with lower velocities and close to the wall the measured velocity is overestimated due to contribution of particles with larger velocities. The velocity measured at the wall will be in fact different from 0 due to contribution of defocused particles, and a non-zero velocity
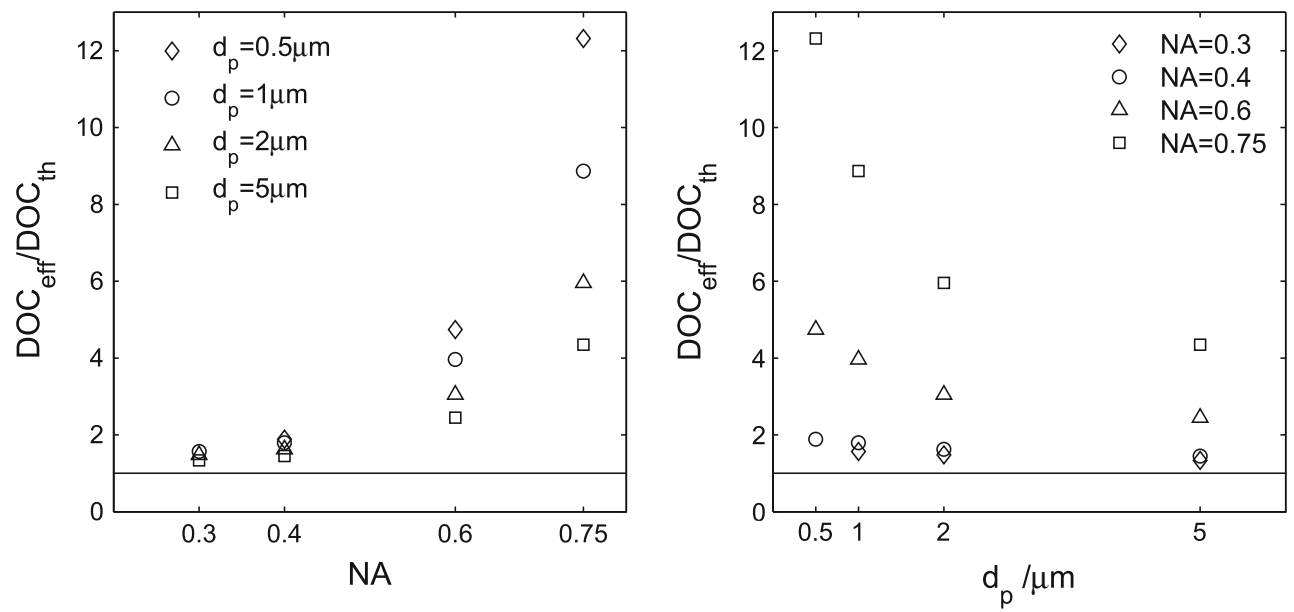

Fig. 9 Effective DOC normalized with the nominal DOC for different combinations of lenses and particle diameters 
will still be measured when the focal plane is moved outside of the channel. Actually, as a consequence of the flattening of the weighting function, the measured velocity is expected to increase again as the focal plane moves away from the channel wall, so that a local minimum of velocity will be present at the $z$ location corresponding to the wall surface. A typical DOC-biased velocity profile is shown in the left graph of Fig. 10.

The velocity $V(0)$ measured at the wall directly relates to the size of the DOC, as shown in the right graph of Fig. 10. This approach can be in principle used as an indirect quantification of the DOC in operative conditions. This is also useful to test the effectiveness of image preprocessing in reducing the bias error in conventional $\mu$ PIV measurements. However, it has to be noted that the bias error cannot be uniquely assigned to the DOC, since it also depends, to a minor extent, on other contributions such as the finite size of the interrogation windows (Keane and Adrian 1990; Westerweel 2000), or the tracer particle concentration, which is lower in the region very close to the wall.

The velocity profiles in a rectangular microchannel with Poiseuille flow were measured using different combinations of lenses and particle sizes, in particular with $20 \times 1$ $0.4,40 \times / 0.6,63 \times / 0.75$ and $d_{p}=0.5,1,2,5 \mu \mathrm{m}$. The velocity profiles were taken in the center of the channel (0.5 times the width), scanning in the z-direction. Results are presented in Fig. 11. For each set of data, the measured velocity profiles were obtained from raw and preprocessed images, and the results were compared with the theoretical profile obtained using nominal ( $V_{\text {th }}$, dotted line) and effective DOC ( $V_{\text {eff }}$, continuous line).

In the graph of Fig. 12, the measured velocity at the wall, presented as a function of the NA and the $d_{p}$, is reported. The velocity values are reported normalized over the theoretical values obtained with nominal (gray markers) and effective DOC (black markers). It is apparent from the graphs that the bias error predicted using the nominal DOC strongly underestimates the actual error, whereas using the effective DOC the prediction shows an excellent match with the experimental results. In particular, for small particles $\left(d_{p}=0.5\right.$ and 1$)$, the ratio between measured and predicted velocity at the wall is always below 1.3 , whereas for larger particles, it varies between 1.5 and 2 . This difference can be also ascribed to the additional bias error introduced by the lower particle concentration close to the wall, which is also proportional to the particle size.

The velocity measurements confirm that the actual DOC in a practical $\mu$ PIV application can be significantly larger than the one predicted using only the nominal NA provided by the manufacturer of the lens. The measurements confirm also that using an effective DOC, calculated using an effective NA experimentally determined from the curvature of the particle image autocorrelation, provides a good estimate of the actual size of DOC.

Finally, the effect of image preprocessing was investigated. Image preprocessing is widely used in practical application of $\mu$ PIV to remove background noise and could in principle eliminate the bias error due to DOC when all out-of-focus particles were removed. To quantify this last effect, we introduce the parameter $C_{p}$ defined as the ratio between the measured velocity at the wall with $\left(V_{\text {prep }}(0)\right)$ and without $(V(0))$ image preprocessing. $C_{p}=0$ means that the image preprocessing was able to completely remove the bias error, whereas $C_{p}=1$ means that the bias error was not diminished at all. Velocity profiles measured using preprocessed images are reported in Fig. 11. In Fig. 13, the $C_{p}$ as a function of the $\mathrm{DOC}_{\text {eff }}$ is reported. The results show that the contribution of image preprocessing is quite limited. In general, it helps to reduce the error by a factor between 0.6 and 1, showing slightly better performance for small DOCs, but it is never able to produce data in which the effect of DOC could be completely neglected. This can be explained by the fact that particles remain
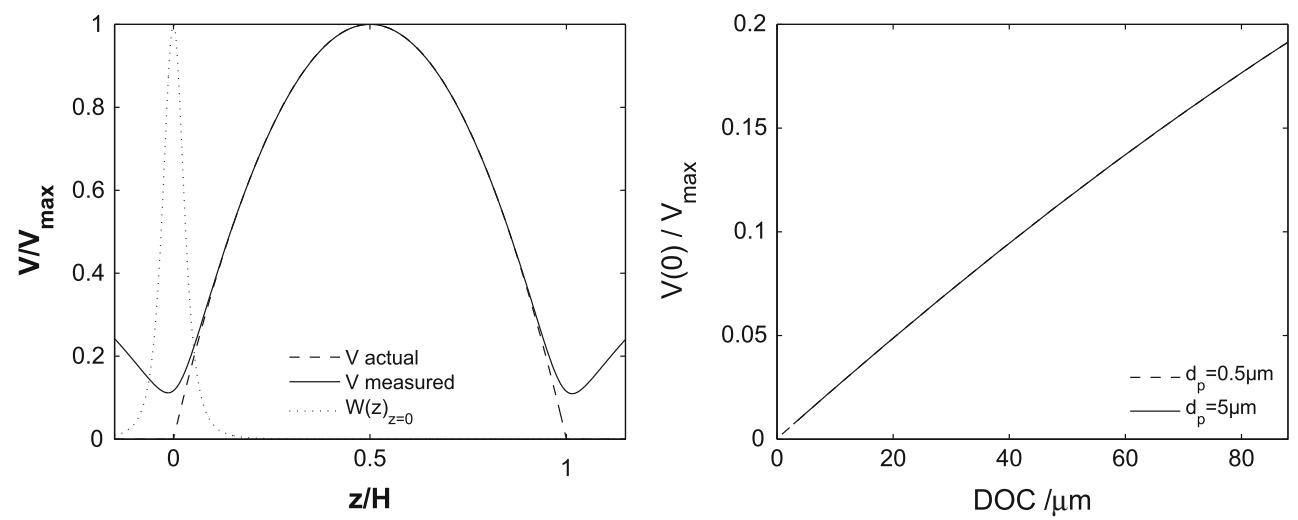

Fig. 10 Theoretical estimation of a measured velocity profile obtained scanning through the $z$-direction with a DOC-biased $\mu$ PIV system (on the left). Bias error at the wall (non-zero velocity) as a function of the DOC of the $\mu$ PIV system (on the right) 
(a)

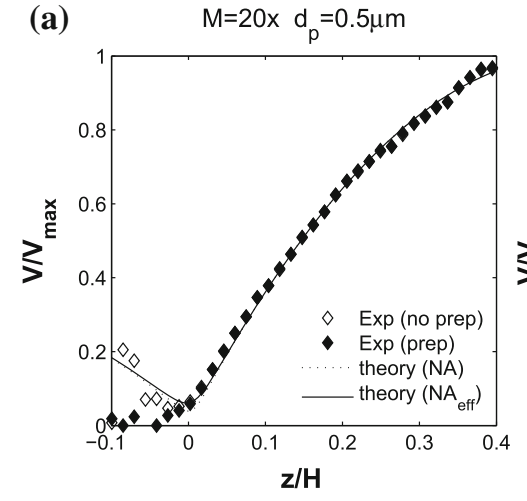

(b)

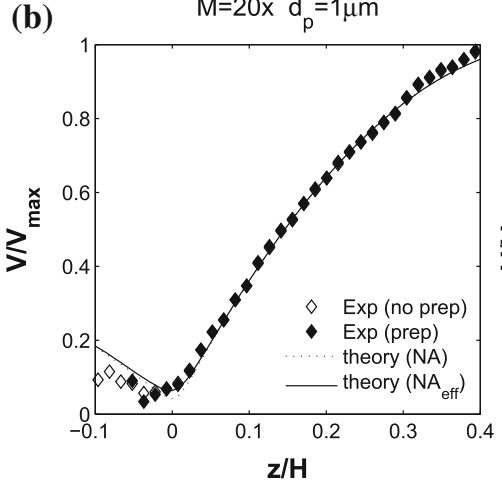

(c)

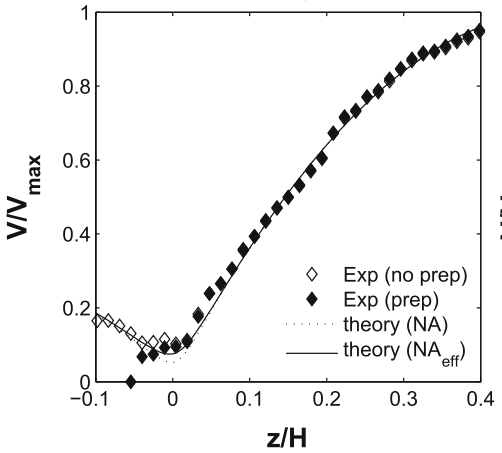

(d)

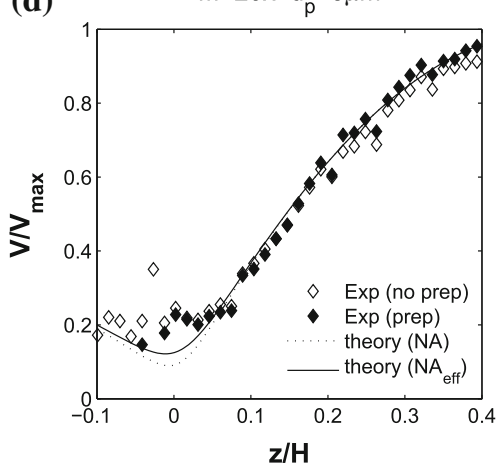

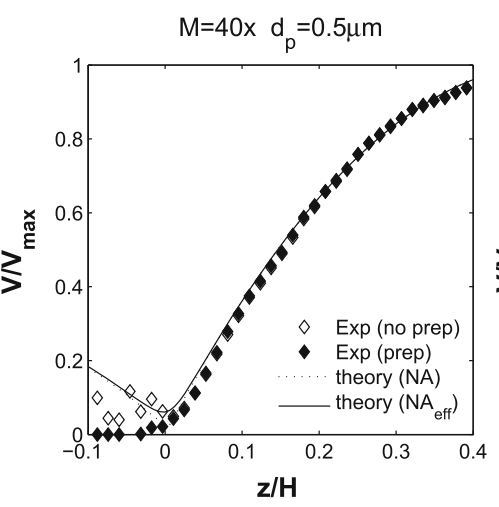
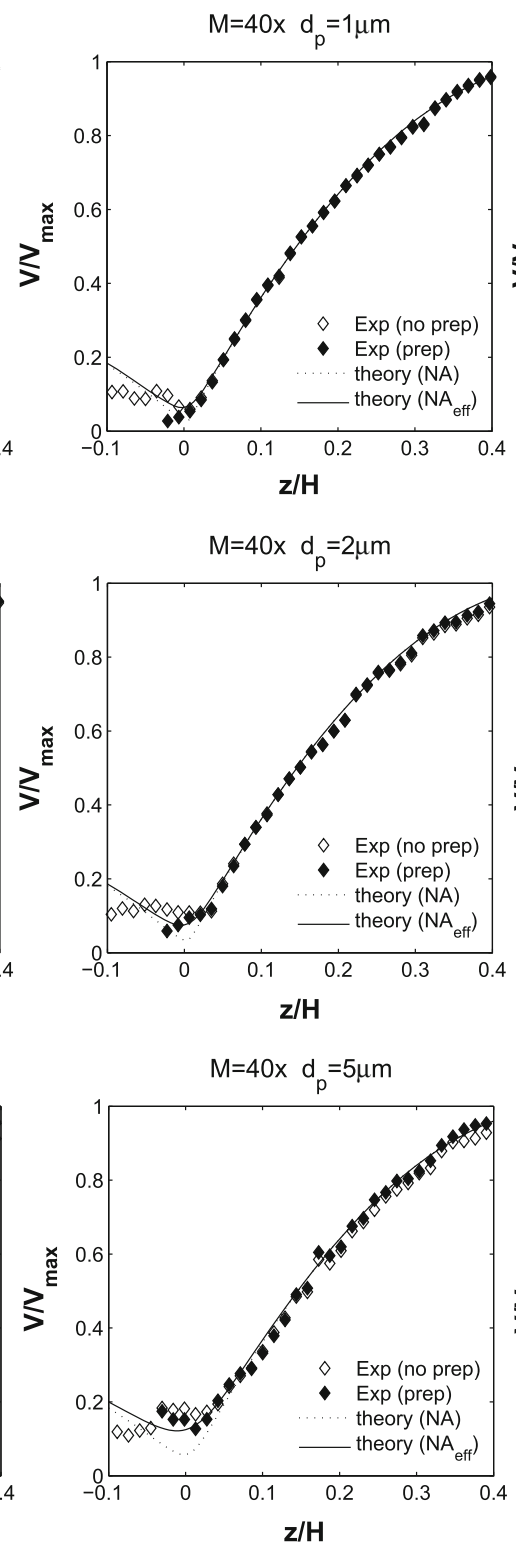
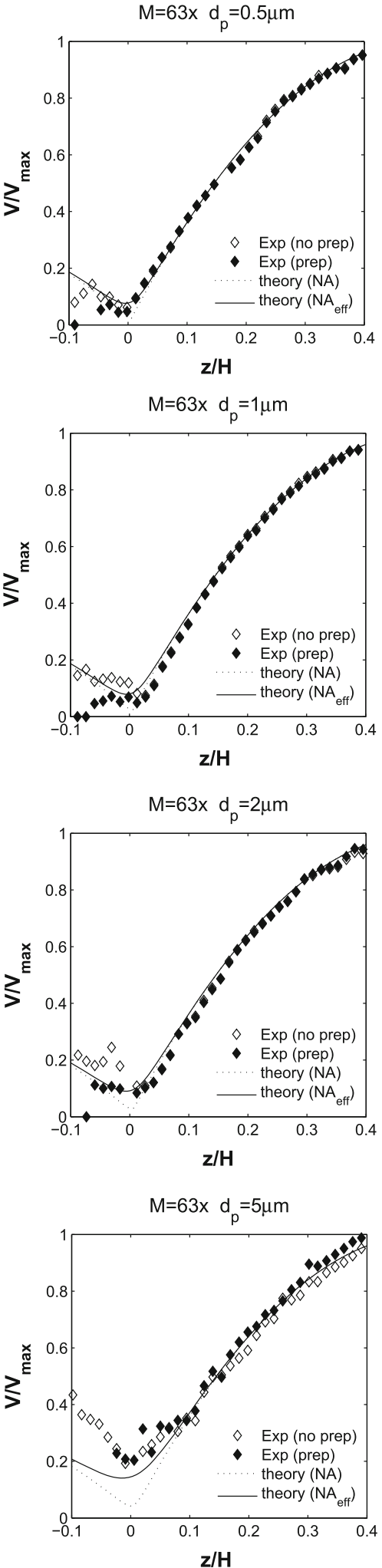

Fig. 11 Comparison of the measured velocity profiles obtained with and without image preprocessing, with the theoretical velocity estimated using nominal (dotted line) and effective (continuous line)
DOC. The velocities correspond to Poiseuille flow in a microchannel and are taken using different combinations of lenses and particle sizes 

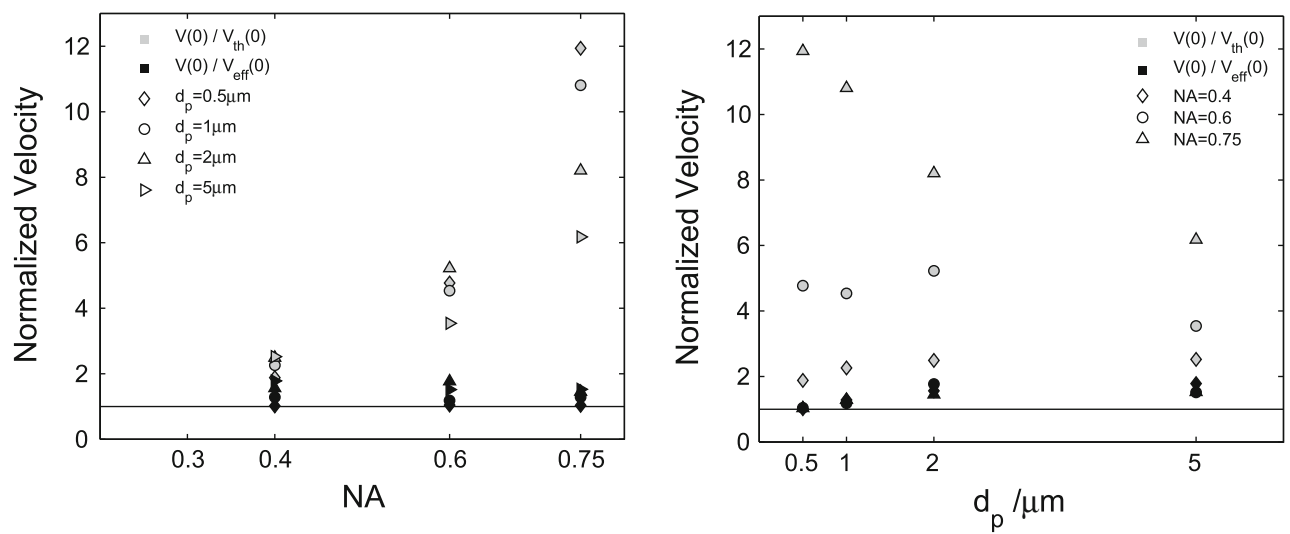

Fig. 12 Velocity measured at the wall normalized over the corresponding theoretical prediction made using nominal and effective DOC. Velocities are reported for different combinations of lenses and particle sizes

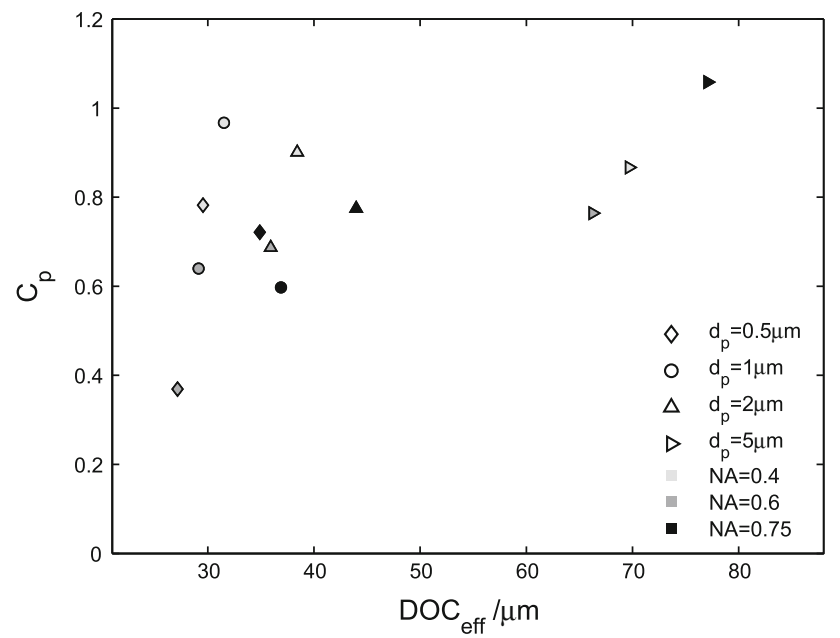

Fig. 13 Ratio between the velocity measured with and without image preprocessing as a function of the effective DOC

in-focus, with a nearly constant diameter and intensity, in a relatively large region, corresponding approximately to $W>0.8$. This prevents the preprocessing algorithm to discriminate and reject particles in that region, so that a bias error due to DOC will be always present.

The $C_{p}$ can be used to estimate the effective DOC achieved after image preprocessing is applied. In fact, as a first approximation, the velocity at the wall can be considered linearly proportional to the DOC (see right graph on Fig. 10). Under this approximation, the $C_{p}$ represents also the ratio between the DOC with and without the image preprocessing and can be directly multiplied in front of Eq. 16.

In conclusion, this experimental analysis shows that is very difficult to get rid of the bias error due to DOC in practical $\mu \mathrm{PIV}$ applications. Increasing the NA of the objective lens does not necessarily correspond to a significantly smaller DOC, since the effective NA resulting at the CCD sensor can be significantly smaller than expected.
The image preprocessing scheme used in this work could only marginally reduce the DOC. Other solutions could be trying different image preprocessing strategies, such the power filter suggested by Bourdon et al. (2004a, b). However, on the opinion of the authors to completely avoid the bias error due to the DOC, other measurement techniques, such as 3D particle tracking methods, should be applied. For instance, Cierpka et al. (2010, 2011a, b) recently showed a method based on astigmatic aberrations that is able to measure 3D velocity fields in microflows without bias error due to the DOC. The effectiveness of this technique has been already proven in comparison with conventional and stereoscopic $\mu$ PIV on a backward-facing step (Cierpka et al. 2011a, b).

\section{Conclusions}

In this article, it was shown that the actual size of DOC in practical $\mu$ PIV experiments can be significantly underestimated when its value is calculated using the nominal NA of the microscope objective lenses. This is particularly evident when high NA objective lenses are used, as shown by experiments. Using an objective lens with $\mathrm{NA}=0.6$, the actual DOC was found to be about 3-5 times larger than the nominal value, depending on size of tracer particles. Using NA $=0.75$, this ratio ranged between 4 and 12 . To account for this problem, it was proposed to use an effective NA to estimate the depth of correlation, experimentally determined from the curvature of the particle image autocorrelation. Measurements in a Poiseuille flow in a microchannel, performed with different objective lenses and tracer particle sizes, demonstrated that using this approach it was possible to accurately predict the bias error at the wall, which is directly related to the actual DOC of the experiment. Furthermore, the effect of image preprocessing on the actual size of DOC was studied. Using a 
preprocessing scheme based on subtracting the sliding minimum over time and an intensity histogram filter, it was possible to obtain a reduction of the DOC on average equal to 0.8 .

Thus, to correctly estimate the actual DOC in a practical $\mu$ PIV experiment, it is here proposed to use an effective DOC given by following expression:

$$
\begin{aligned}
\mathrm{DOC}_{\mathrm{eff}}= & C_{p} \frac{n_{w}}{n_{0}}\left\{\frac{(1-\sqrt{\varepsilon})}{\sqrt{\varepsilon}}\left(\frac{n_{0}^{2}}{\mathrm{NA}_{\mathrm{eff}}^{2}}-1\right)\right. \\
& \left.\times\left[d_{p}^{2}+1.49 \lambda^{2}\left(\frac{n_{0}^{2}}{\mathrm{NA}_{\mathrm{eff}}^{2}}-1\right)\right]\right\}^{1 / 2}
\end{aligned}
$$

where $C_{p}$ is an experimental parameter accounting for the image preprocessing (typically between 0.6 and 1) and $\mathrm{NA}_{\text {eff }}$ is an effective numerical aperture equal to $C_{d} \mathrm{NA}$, where NA is the nominal numerical aperture of the microscope objective lens and $C_{d}$ is an experimental parameter determined from the curvature of the particle image autocorrelation.

Finally, the experimental analysis carried out in this work suggests that completely removing the bias error due to DOC in practical $\mu$ PIV applications is very difficult or not possible at all. This is due to the large effective DOC obtained even with high NA objectives and to the limited efficacy of preprocessing algorithm to reduce this error. If measurements without bias error from DOC were necessary for a specific application, the authors advise to employ different measurement techniques, such as 3D PTV methods.

Open Access This article is distributed under the terms of the Creative Commons Attribution Noncommercial License which permits any noncommercial use, distribution, and reproduction in any medium, provided the original author(s) and source are credited.

\section{References}

Adrian RJ (1991) Particle-imaging techniques for experimental fluid mechanics. Annu Rev Fluid Mech 23:261-304

Adrian RJ, Yao CS (1985) Pulsed laser technique application to liquid and gaseous flows and the scattering power of seed materials. Appl Optics 24(1):44-52
Bourdon CJ, Olsen MG, Gorby AD (2004a) Power-filter technique for modifying depth of correlation in micropiv experiments. Exp Fluids 37(2):263-271

Bourdon CJ, Olsen MG, Gorby AD (2004b) Validation of an analytical solution for depth of correlation in microscopic particle image velocimetry. Meas Sci Technol 15(2):318-327

Bourdon CJ, Olsen MG, Gorby AD (2006) The depth of correlation in micro-PIV for high numerical aperture and immersion objectives. J Fluid Eng-T ASME 128(4):883-886

Cierpka C, Segura R, Hain R, Kähler CJ (2010) A simple single camera $3 \mathrm{C} 3 \mathrm{D}$ velocity measurement technique without errors due to depth of correlation and spatial averaging for microfluidics. Meas Sci Technol 21(4):045401

Cierpka C, Rossi M, Segura R, Kähler CJ (2011a) On the calibration of astigmatism particle tracking velocimetry for microflows. Meas Sci Technol 22(1):015401

Cierpka C, Rossi M, Segura R, Mastrangelo F, Kähler CJ (2011b) A comparative analysis of the uncertainty of astigmatism- $\mu \mathrm{PTV}$, stereo- $\mu$ PIV, and $\mu$ PIV. Exp Fluids doi:10.1007/s00348-0111075-5

Keane RD, Adrian RJ (1990) Optimization of particle image velocimeters. Part I: double pulsed systems. Meas Sci Technol 1(11):1202-1215

Kloosterman A, Poelma C, Westerweel J (2011) Flow rate estimation in large depth-of-field micro-piv. Exp Fluids 50:1587-1599

Lindken R, Rossi M, Grosse S, Westerweel J (2009) Micro-particle image velocimetry ( $\mu \mathrm{PIV})$ : recent developments, applications, and guidelines. Lab Chip 9(17):2551-2567

Meinhart CD, Wereley ST (2003) The theory of diffraction-limited resolution in microparticle image velocimetry. Meas Sci Technol 14(7): 1047-1053

Meinhart CD, Wereley ST, Gray MHB (2000) Volume illumination for two-dimensional particle image velocimetry. Meas Sci Technol 11(6):809-814

Olsen MG (2009) Directional dependence of depth of correlation due to in-plane fluid shear in microscopic particle image velocimetry. Meas Sci Technol 20(1):015402

Olsen MG, Adrian RJ (2000) Out-of-focus effects on particle image visibility and correlation in microscopic particle image velocimetry. Exp Fluids 29:S166-S174

Raffel M, Willert CE, Wereley ST, Kompenhans J (2007) Particle image velocimetry: a practical guide. Springer, Berlin

Rossi M, Lindken R, Westerweel J (2010) Optimization of multiplane $\mu$ PIV for wall shear stress and wall topography characterization. Exp Fluids 48(2):211-223

Santiago JG, Wereley ST, Meinhart CD, Beebe DJ, Adrian RJ (1998) A particle image velocimetry system for microfluidics. Exp Fluids 25(4):316-319

Westerweel J (2000) Theoretical analysis of the measurement precision in particle image velocimetry. Exp Fluids 29:S3-S12

Yeo TTE, Ong SH, Jayasooriah, Sinniah R (1993) Autofocusing for tissue microscopy. Image Vision Comput 11(10):629-639 